\title{
Percutaneous fixation of traumatic pubic symphysis diastasis using a TightRope and external fixator versus using a cannulated screw
}

Yongzeng Feng ${ }^{1}$, Jianjun Hong ${ }^{2}$, Xiaoshan Guo ${ }^{2}$, Chuangxin Lin ${ }^{3}$, Wei Ling ${ }^{1}$, Lifeng Zhang ${ }^{1}$ and Gang Wang ${ }^{1 *}$

\begin{abstract}
Background: The aim of the study was to introduce a new percutaneous technique for the treatment of traumatic pubic symphysis diastasis using a TightRope and external fixator. A comparison between this technique and percutaneous fixation using a cannulated screw was performed.

Methods: From January 2009 to December 2013, 26 patients with type II traumatic pubic symphysis diastasis were treated at two level 1 regional trauma centers. Among them, 10 patients were treated with a percutaneous TightRope and external fixator and 16 patients were treated with percutaneous cannulated screw fixation. Functional outcomes were evaluated using the Majeed scoring system. Patient satisfaction was evaluated using the modified visual analog scale. Radiological results were assessed based on the width of pubic symphysis preoperatively, immediately postoperatively, and at the final follow-up. Postoperative complications were also recorded.

Results: There were no significant differences between the groups in Majeed scores and patient satisfaction $(p>0$. 05). There were no significant differences in the width of pubic symphysis preoperatively, immediately postoperatively, and at the final follow-up ( $p>0.05)$. No significant differences were found regarding infection, fixation failure, or the need for revision surgery $(p>0.05)$.

Conclusions: The new percutaneous technique using a TightRope and external fixator is a successful alternative for the treatment of type II traumatic pubic symphysis diastasis, which results in similar outcomes comparing to percutaneous cannulated screw fixation.
\end{abstract}

Keywords: Symphysis pubis diastasis, Percutaneous reduction, TightRope fixation, Cannulated screw, External fixation, Comparative study

\section{Background}

Pubic symphysis diastasis (PSD) is typically associated with a high-energy mechanism of injury [1]. Although many treatments have been reported on restoration of the anatomy of pubic symphysis, the appropriate strategy remains controversial $[2,3]$.

\footnotetext{
* Correspondence: 13858878561@163.com

'Department of Orthopaedic Trauma, Nanfang Hospital, Southern Medical University, No.1838, Guangzhou Road, Guangzhou, Guangdong Province 510000, China

Full list of author information is available at the end of the article
}

PSD is often a result of anterior-posterior compression (APC) injury based on the Young and Burgess classification system. The injuries are classified into three types, i.e., APC-I (slight widening of pubic symphysis or anterior sacroiliac joint, or both); APC-II (widened anterior sacroiliac joint $>2.5 \mathrm{~cm}$, disrupted anterior sacroiliac, sacrotuberous, and sacrospinous ligaments, intact posterior sacroiliac ligaments); and APC-III (complete sacroiliac joint disruption with lateral displacement, disrupted anterior sacroiliac, sacrotuberous, and disrupted posterior sacroiliac ligaments) [4-6]. 
APC-II injuries are rotationally unstable. Open reduction and internal fixation using plate and screw system has been widely accepted as the standard treatment $[7,8]$. However, the technique needs extensive exposure of the pubic symphysis, resulting in more complications, such as blood loss, neural and vascular injuries, wound problems, and heterotopic bone formation [9-11]. In addition, the physiological movement across the pubic symphysis often leads to fixation failure [12].

In order to reduce the complications, several other techniques have been reported, including external pelvic fixation, percutaneous cannulated screw fixation, minimally invasive plate osteosynthesis, and Endobutton technique for dynamic fixation [2-6]. In our hospital, percutaneous cannulated screw fixation is the common surgical procedure in the past 10 years. Since 2009, we have performed a new percutaneous dynamic fixation technique for PSD using a TightRope (Arthrex, Naples, FL, USA) device and external fixator (Fig. 1).

The purpose of the study was to introduce the new percutaneous technique for the treatment of APC-II traumatic PSD using a TightRope and external fixator. In order to better understand the procedure, we performed a comparison between this technique and percutaneous fixation using a cannulated screw [13].

\section{Methods}

The study was approved by the Ethical Review Boards of Nanfang Hospital, Southern Medical University, and the Second Affiliated Hospital of Wenzhou Medical University. Informed consent for participation was obtained from all the patients.

From January 2009 to December 2013, a total of 35 consecutive patients with APC-II traumatic PSD were treated at two level 1 regional trauma centers. Inclusion criteria were adult patients who had a closed APC-II injury. The exclusion criteria were as follows: an APC-I
PSD was excluded because the injury is often treated conservatively; an APC-III injury was excluded because combined posterior fixation is needed; patients who had lateral compression, vertical shear or combined mechanism fractures, open fractures, combined acetabular fractures, or pubic rami fractures were excluded; patients who had immature skeletons or medical contraindications, such as combined neurovascular injuries or diabetes, were excluded. Thus, 26 patients were included in the study and randomly allocated to group A (percutaneous fixation using a TightRope and external fixator) and group B (percutaneous fixation using a cannulated screw). The operator and tester were blinded.

Preoperatively, radiographs and CT scans were obtained to assess patterns of the injuries (Fig. 2a, b). Three patients underwent early pelvic angiography and embolization to stabilize their blood pressure.

\section{Surgical techniques \\ Group A}

The patient was placed in the supine position, and skin preparation was done. Two Schanz pins were inserted into either side of the iliac crest. Under C-arm fluoroscopic monitoring, the symphysis was reduced by manipulating the pins, and reduction was maintained percutaneously using pointed reduction clamps applying on the pubic tubercles (Fig. 2c). Utilizing a matching drill guide, the insertion and exertion points were located at the juncture between the pubic tubercle and obturator on either side (Fig. 2d). A 1.5-mm guide wire was drilled transversally across the symphysis. Correct wire positioning was confirmed under anteroposterior, outlet, and inlet views. Care was taken not to injure the spermatic cord in male patients and the round ligament of uterus in female patients. A bony tunnel was created using a $4.0-\mathrm{mm}$ cannulated drill bit introduced over the guide wire (Fig. 3a). Then, a 3.0-mm hollow tube was inserted through the drilled tunnel, in which a traction

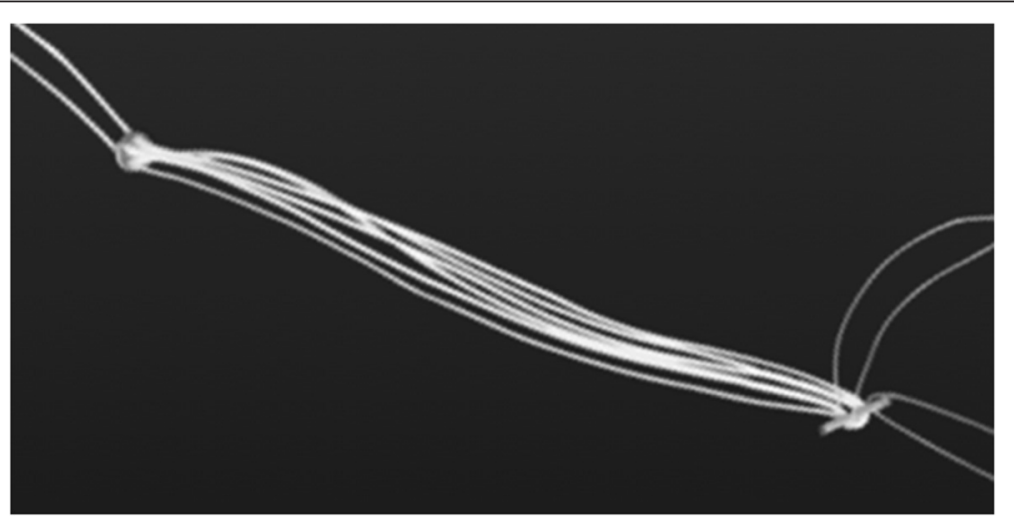

Fig. 1 TightRope (Arthrex) device consists of a circular-shaped button and an oblong-shaped button. The buttons are connected with FiberWire threads 

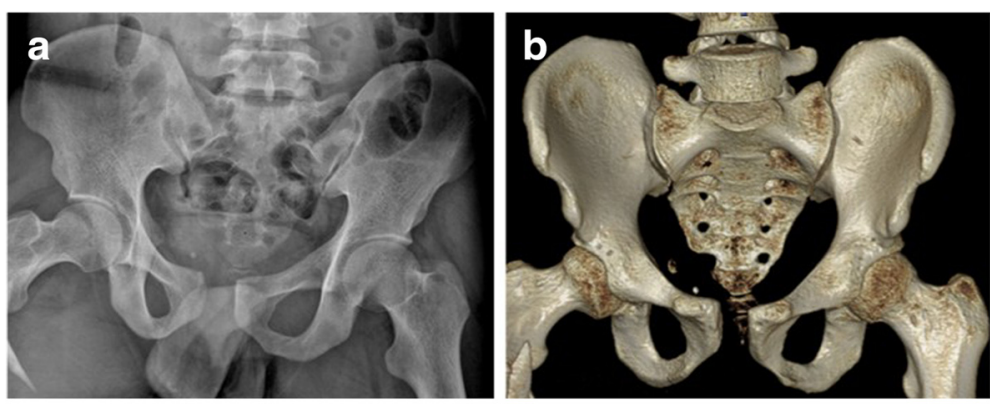

C

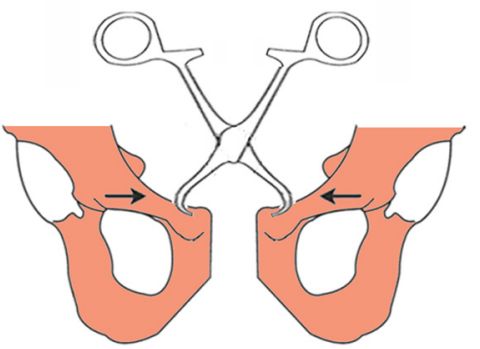

d

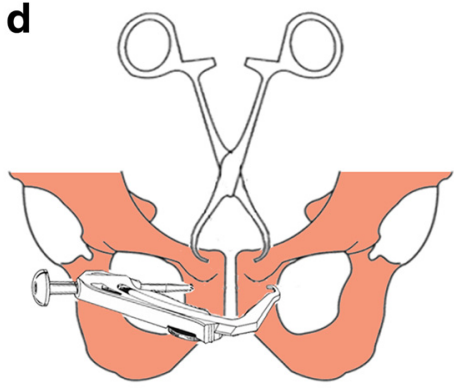

Fig. 2 A male patient suffered pubic symphysis diastasis in a road traffic accident. a Preoperative anteroposterior radiograph. b Three-dimensional CT. c Reduction is maintained using pointed reduction clamps. $\mathbf{d}$ The insertion and exertion of the guide wire are located using a drill guide
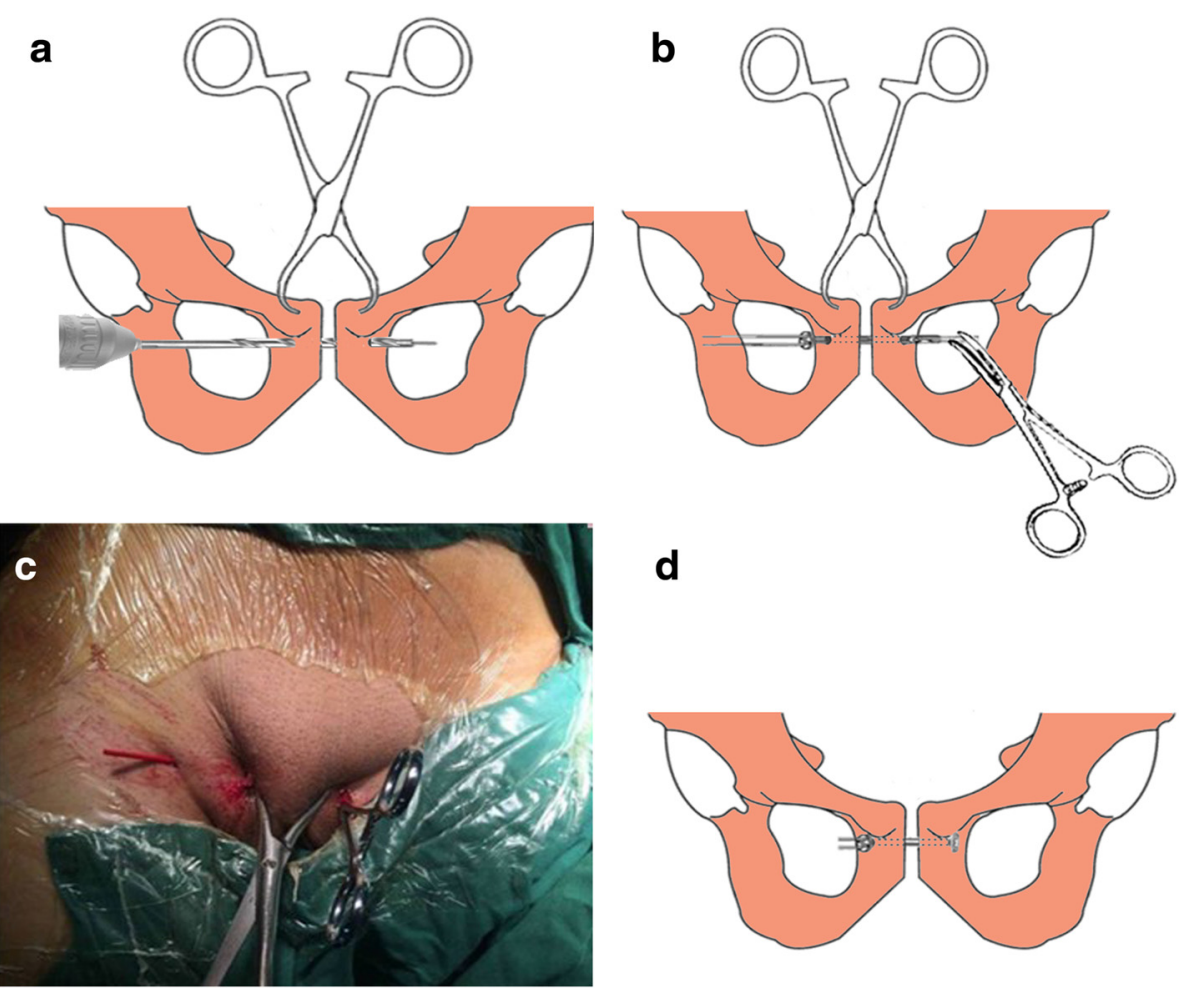

d

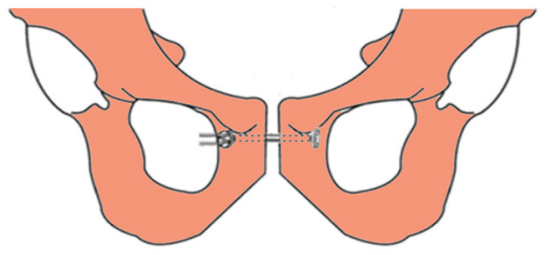

Fig. 3 a A tunnel is created using a cannulated drill bit introduced over the guide wire. $\mathbf{b}$ The oblong-shaped button is passed through the tunnel. c A hollow tube was used across the traction thread. $\mathbf{d}$ The free ends of the FiberWire suture are tightened 
thread was introduced in advance. The oblong button of TightRope was passed through the drilled tunnel to the opposite side by pulling the traction thread using a hemostat (Fig. 3b). The tube was removed, and both buttons were seated flush in the correct position (Fig. 3c). The FiberWire suture at the skin insertion was tightened manually (Fig. 3d). Finally, an external fixator was fixed on the Schanz pins to reinforce pelvic stability.

\section{Group B}

As described by Chen et al. [3], the symphysis was stabilized percutaneously using a $6.5-\mathrm{mm}$ cannulated lag screw. No additional external fixation was used.

\section{Postoperative managements}

Patients were encouraged to perform non-weightbearing exercises when pain could be tolerated. The external fixator in group A was removed 6 weeks after surgery. Partial weight bearing was allowed at that time and progressed gradually according to the condition of each patient's associated injuries. All patients were followed up at 6 weeks, 3 months, 6 months, and 12 months postoperatively and annually thereafter (Fig. 4).

\section{Outcome evaluation}

Functional outcomes were evaluated using the scoring system described by Majeed [14], including pain, sitting, sexual intercourse, walking, and work. Patient satisfaction was evaluated using the modified visual analog scale based on a range from 0 to 10 , with 0 indicating maximum dissatisfaction and 10 indicating full satisfaction. Postoperative complications were also recorded.

The width of the pubic symphysis was measured preoperatively, immediately after surgery, and at the final follow-up using the picture archiving and communication system (Infinitt, Seoul, South Korea) [15]. Fixation failure was defined as loosening or breakage of Endobutton device or screw.

\section{Statistical analysis}

Statistical analysis was performed with SPSS 17.0 software for Windows. Continuous data with a normal distribution were expressed as the mean \pm standard deviation. Continuous variables with non-normal distributions were analyzed with the Mann-Whitney $U$ test. Non-paired $t$ test was used for comparison of continuous data that appeared to be approximately normally distributed. Categorical data were statistically analyzed using Fisher's exact test $(n<40$ or $t<1)$. A $p$ value of $<0.05$ was considered statistically significant.

\section{Results}

All 26 patients were followed for an average of 15 months (range, 12-20 months). We found no significant differences in patient age, sex, injury mechanism, and time from injury to operation between the two groups $(p>0.05)$. The operative time of group A was $48.5 \pm 9.4 \mathrm{~min}$, which was significantly longer than that of group B $(27.3 \pm 5.1 \mathrm{~min})(p<0.001$; Table 1$)$. One patient in group A and two patients in group $B$ had urethral injuries. As a result, one patient was treated conservatively and two other patients underwent surgery. Two patients in group $\mathrm{A}$ and three patients in group B had cerebral contusions or thoracic injuries, or both, and all of them were treated conservatively. Four patients in group A had combined femoral $(n=2)$, tibiofibular $(n=1)$, and lumbar $(n=1)$ fractures. Five patients in group B had combined femoral $(n=3)$, forearm $(n=$ $1)$, and lumbar $(n=1)$ fractures. Those fractures were simultaneously treated with open or closed reduction and internal fixation, and bone healing was achieved in all cases.

Preoperatively, the width of pubic symphysis was $40.0 \pm$ $12.3 \mathrm{~mm}$ in group A and $41.4 \pm 10.4 \mathrm{~mm}$ in group B. Immediately after surgery, the width decreased to $4.1 \pm 0.6$ and $4.5 \pm 0.7 \mathrm{~mm}$ in groups $\mathrm{A}$ and $\mathrm{B}$, respectively $(p<$ 0.001 ). At the final follow-up, the width was $4.3 \pm 0.5$ and $4.7 \pm 0.7 \mathrm{~mm}$, respectively. In both groups, the width of pubic symphysis was significantly decreased immediately

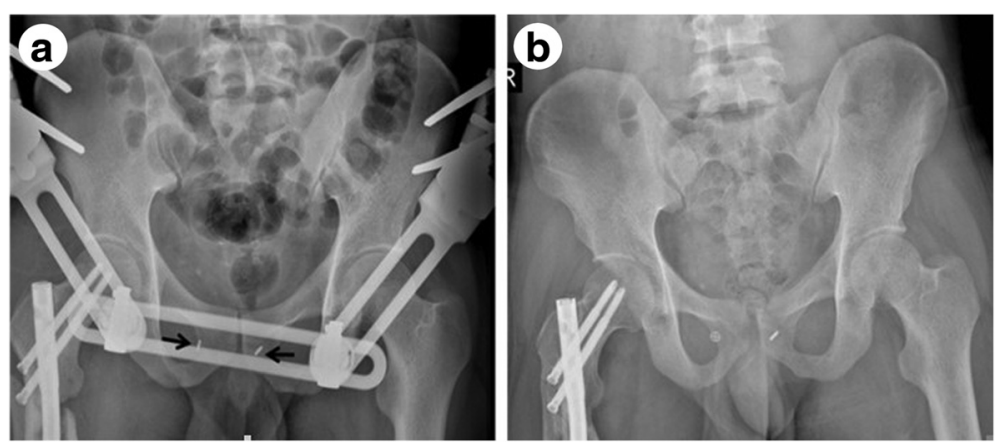

Fig. 4 Postoperative radiographs. a Immediately after surgery. b One year after surgery 
Table 1 Patient characteristics and surgical details of the two groups $(x \pm s)$

\begin{tabular}{|c|c|c|c|}
\hline & $\begin{array}{l}\text { Group A } \\
(n=10)\end{array}$ & $\begin{array}{l}\text { Group B } \\
(n=16)\end{array}$ & $p$ value \\
\hline Age (year) & $32.5 \pm 6.2$ & $33.2 \pm 5.8$ & 0.781 \\
\hline $\operatorname{Sex}(m / f)$ & $7 / 3$ & $11 / 5$ & 1.000 \\
\hline Injury mechanism ( $n$ ) & & & 1.000 \\
\hline Traffic accident & 8 & 13 & \\
\hline Crushing & 2 & 3 & \\
\hline Time to operation (day) & $4.8 \pm 2.9$ & $4.1 \pm 3.0$ & 0.580 \\
\hline Operative time (min) & $48.5 \pm 9.4$ & $27.3 \pm 5.1$ & 0.000 \\
\hline \multicolumn{4}{|l|}{ Width of pubic symphysis (mm) } \\
\hline Preoperation & $40.0 \pm 12.3$ & $41.4 \pm 10.4$ & 0.813 \\
\hline Immediately postoperation & $4.1 \pm 0.6^{*}$ & $4.5 \pm 0.7^{* *}$ & 0.118 \\
\hline Final follow-up & $4.3 \pm 0.5^{* * *}$ & $4.7 \pm 0.7^{* * * *}$ & 0.113 \\
\hline \multicolumn{4}{|l|}{ Complications ( $n$ ) } \\
\hline Infection & 1 & 0 & 0.385 \\
\hline Fixation failure & 1 & 3 & 1.000 \\
\hline Revision surgery & 0 & 1 & 1.000 \\
\hline Satisfaction (MVAS) & $8.1 \pm 1.2$ & $8.4 \pm 1.0$ & 0.538 \\
\hline Majeed score $(n)$ & & & 0.897 \\
\hline Excellent (>85) & 7 & 11 & \\
\hline Good (70-84) & 3 & 4 & \\
\hline Fair (55-69) & 0 & 1 & \\
\hline Poor $(<55)$ & 0 & 0 & \\
\hline
\end{tabular}

MVAS modified visual analog scale

${ }^{*} p=0.000 ;{ }^{* *} p=0.000 ;{ }^{* * *} p=0.419 ;{ }^{* * *} p=0.445$

after surgery $(\mathrm{Pa}=0.000, \mathrm{~Pb}=0.000)$, but no significant difference was found between the data measured immediately after surgery and at the final follow-up $(\mathrm{Pc}=0.419$, $\mathrm{Pd}=0.445)$ (Table 1). We found no significant difference between the groups in preoperative or immediately postoperative data, nor data measured at the final follow-up.

One patient in group A developed a superficial infection at the skin insertion. One patient in group A and three patients in group B had a fixation failure. One patient in group B had a revision surgery. No significant difference was found regarding infection, implant failure, or revision surgery between the two groups. We found no significant difference in Majeed scores or patient satisfaction between the groups (Table 1). Detailed results and scores regarding pain, sitting, sexual intercourse, walking, and work are shown in Table 2.

\section{Discussion}

PSD may occur alone or be combined with other pelvic fractures. According to APC in the Young-Burgess classification of pelvic disruption, a diastasis of more than $2.5 \mathrm{~cm}$ is classified as an APC-II injury pattern. It is a common "open book" injury with rotational instability.
It is routinely treated surgically [16]. We found that our percutaneous technique using a TightRope and external fixator is a safe, minimally invasive, dynamic fixation for the treatment of APC-II traumatic PSD.

In adults, the pubic symphysis has small-magnitude, multidirectional movements under physiological conditions $[17,18]$. The traditional static immobilization using a plate and screw system carries a high rate of fixation failure [19]. The reported percentage is above $30 \%$. Implant loosening and breakage may result in the recurrence of PSD and pain [12]. Chen et al. reported percutaneous cannulated screw fixation. Comparing to the plate fixation, the cannulated screw fixation can also well maintain the reduction. The minimally invasive technique has advantages of a lower risk of iatrogenic injuries, more rapid recovery, and better appearance. However, the screw fixation is also a static immobilization, which carries the similar fixation failure [3]. Chen et al. also evaluated 21 patients with PSD undergoing Endobutton (Smith and Nephew, Memphis, TN, USA) fixation. The results indicate that Endobutton fixation might be an alternative treatment for APC-II injuries. However, they used it through open reduction. The length of the loop in Endobutton is specified, and it cannot be tightened freely as needed [6].

In order to decrease the complications, we introduced the dynamic percutaneous fixation technique using a TightRope device. The device was originally designed for stabilization of tibiofibular syndesmosis [20]. It consists of two metal buttons, circular and oblong buttons, joined by a continuous loop of FiberWire suture. It can be tightened manually and does not need to be tied in a knot. It has also been indicated in acromioclavicular dislocation, distal clavicle fractures, and anterior cruciate ligament reconstruction [21-23]. TightRope provides semi-rigid fixation that allows minor physiological motion, which decreases the stress on the implant. The additional external fixation adds more stability in the early postoperative stage.

The best indication of percutaneous TightRope technique might be APC-II injury or type B1 or B2 (according to the Tile classification). Increased clinical experience and biomechanical studies about TightRope may make it easier to use in more complicated cases, such as APC-III injuries. Contraindications are open pelvic fractures, combined acetabular fractures, pubic rami fractures, and medical contraindications.

Advantages of the technique are a minimally invasive procedure, less blood loss, minimal complications, and rapid recovery. The dynamic fixation decreases stress at the symphysis pubis during its ligament healing and micromovement, which decreases the risk of implant failure. Implant removal is not necessary. The disadvantages are a complex procedure, and the operation time is relatively longer. 
Table 2 Detailed scores according to the Majeed scoring system at the final follow-up

\begin{tabular}{|c|c|c|c|c|c|c|c|}
\hline Group/no. & Pain & Sitting & Sexual intercourse & Walking & Work & Total & Grade \\
\hline \multicolumn{8}{|l|}{ A } \\
\hline 1 & 30 & 8 & 4 & 34 & 16 & 92 & Excellent \\
\hline 2 & 30 & 10 & 4 & 36 & 16 & 96 & Excellent \\
\hline 3 & 25 & 8 & 3 & 34 & 16 & 86 & Excellent \\
\hline 4 & 25 & 12 & 3 & 28 & 16 & 84 & Good \\
\hline 5 & 30 & 10 & 4 & 34 & 16 & 96 & Excellent \\
\hline 6 & 20 & 6 & 4 & 28 & 16 & 74 & Good \\
\hline 7 & 25 & 8 & 4 & 32 & 20 & 89 & Excellent \\
\hline 8 & 25 & 6 & 3 & 32 & 16 & 82 & Good \\
\hline 9 & 30 & 10 & 4 & 34 & 20 & 98 & Excellent \\
\hline 10 & 25 & 10 & 4 & 34 & 20 & 93 & Excellent \\
\hline \multicolumn{8}{|l|}{ B } \\
\hline 1 & 25 & 8 & 4 & 32 & 20 & 89 & Excellent \\
\hline 2 & 25 & 10 & 4 & 34 & 20 & 93 & Excellent \\
\hline 3 & 30 & 10 & 4 & 36 & 16 & 96 & Excellent \\
\hline 4 & 20 & 8 & 4 & 34 & 16 & 82 & Good \\
\hline 5 & 25 & 10 & 4 & 32 & 20 & 91 & Excellent \\
\hline 6 & 20 & 6 & 4 & 28 & 16 & 74 & Good \\
\hline 7 & 25 & 8 & 3 & 32 & 12 & 80 & Good \\
\hline 8 & 25 & 10 & 4 & 36 & 20 & 95 & Excellent \\
\hline 9 & 25 & 8 & 4 & 30 & 16 & 83 & Good \\
\hline 10 & 30 & 8 & 4 & 36 & 20 & 98 & Excellent \\
\hline 11 & 20 & 6 & 3 & 28 & 12 & 69 & Fair \\
\hline 12 & 25 & 10 & 4 & 30 & 20 & 89 & Excellent \\
\hline 13 & 30 & 10 & 4 & 34 & 16 & 94 & Excellent \\
\hline 14 & 30 & 10 & 4 & 36 & 12 & 92 & Excellent \\
\hline 15 & 25 & 10 & 4 & 36 & 20 & 95 & Excellent \\
\hline 16 & 25 & 8 & 4 & 36 & 16 & 89 & Excellent \\
\hline
\end{tabular}

The present study has some limitations. The small number of patients weakens the statistical power of the final results. Further investigation in a larger sample size and longer follow-up time is needed to obtain more overall clinical data. In addition, the differences in surgeons' performance and patients' associated injuries may have decreased the generalization power of this study. Furthermore, biomechanical research on the technique is necessary. Despite all these limitations, the present study contributes significantly to the current treatment of pubic symphysis diastasis.

\section{Conclusions}

The dynamic percutaneous technique using a TightRope and external fixator is a minimally invasive procedure with minimal morbidity. Both the TightRope device and the cannulated screw are effective in the treatment of APC-II traumatic PSD.
Acknowledgements

The authors would like to thank Dr. Xiaolong Shui for his help with this study. This research was performed at the Department of Orthopaedic Trauma, Nanfang Hospital, Southern Medical University, No. 1838,

Guangzhou Road, Guangzhou, Guangdong Province, China, and Department of Orthopaedics Surgery of the Second Affiliated Hospital of Wenzhou Medical University, No. 109, Xue Yuan West Road, Wenzhou, Zhejiang Province, China.

\section{Funding}

This research was supported by the Zhejiang Provincial Traditional Chinese Medicine Technology project funding (No. 2016ZA141) and Zhejiang Provincial Medical and Health Technology Project funding (No. 2016KYA138). The funders had no role in the study design, data collection or analysis, decision to publish, or preparation of the manuscript.

\section{Authors' contributions}

YZF, JJH, and GW designed the study. YZF, XSG, and CXL obtained the funding and/or ethics approval. YZF, CXL, and WL collected the data. YZF, WL, and LFZ analyzed the data. YZF, XSG, and GW interpreted the data. YZF, $J \mathrm{JH}$, and GW composed the article. All authors read and approved the final manuscript. 


\section{Competing interests}

The authors declare that they have no competing interests.

\section{Ethics approval and consent to participate}

The study was approved by the Ethical Review Boards of Nanfang Hospital, Southern Medical University (Guangzhou, Guangdong, China), and the Second Affiliated Hospital of Wenzhou Medical University (Wenzhou, Zhejiang, China) and was performed in accordance with the ethical standards of the Declaration of Helsinki of 1964.

\section{Author details}

'Department of Orthopaedic Trauma, Nanfang Hospital, Southern Medical University, No.1838, Guangzhou Road, Guangzhou, Guangdong Province 510000, China. ${ }^{2}$ Department of Orthopaedics Surgery, The Second Affiliated Hospital of Wenzhou Medical University, No.109, Xue Yuan West Road, Wenzhou, Zhejiang Province 325027, China. ${ }^{3}$ Department of Orthopaedics Surgery, The Third Affiliated Hospital of Southern Medical University, No.183, Zhongshan Road, Guangzhou, Guangdong Province 510000, China.

\section{Received: 26 February 2016 Accepted: 11 May 2016}

\section{Published online: 27 May 2016}

\section{References}

1. Putnis SE, Pearce R, Wali UJ, Bircher MD, Rickman MS. Open reduction and internal fixation of a traumatic diastasis of the pubic symphysis: one-year radiological and functional outcomes. J Bone Joint Surg Br. 2011:93:78-84

2. Bircher MD. Indications and techniques of external fixation of the injured pelvis. Injury. 1996:27 Suppl 2:B3-19.

3. Chen L, Zhang G, Song D, Guo X, Yuan W. A comparison of percutaneous reduction and screw fixation versus open reduction and plate fixation of traumatic symphysis pubis diastasis. Arch Orthop Trauma Surg. 2012;132: 265-70.

4. Yu KH, Hong JJ, Guo XS, Zhou DS. Comparison of reconstruction plate screw fixation and percutaneous cannulated screw fixation in treatment of Tile B1 type pubic symphysis diastases: a finite element analysis and 10-year clinical experience. J Orthop Surg Res. 2015;10:151

5. Liu Z, Wang K, Zhang K, Zhou J, Zhang Y. Minimally invasive surgery (MIS) of anterior ring fracture combined with pubic symphysis separation. Med Sci Monit. 2014;20:1913-7.

6. Chen L, Ouyang Y, Huang G, Lu X, Ye XS, Hong J. Endobutton technique for dynamic fixation of traumatic symphysis pubis disruption. Acta Orthop Belg. 2013:79:54-9.

7. Varga E, Hearn T, Powell J, Tile M. Effects of method of internal fixation of symphyseal disruptions on stability of the pelvic ring. Injury. 1995:26:75-80.

8. Fr S, Schwappach JR, Routt Jr ML, Agnew SG, Harrington RM, Tencer AF. Evaluation of new plate designs for symphysis pubis internal fixation. J Trauma. 1996:41:498-502.

9. Pernal GE, Tile M, Wadell JP, Garside H. Pelvic disruption: assessment and classification. Clin Orthop Relat Res. 1980;151:12-21.

10. Matta JM. Indications for anterior fixation of pelvic fractures. Clin Orthop Relat Res. 1996;329:88-96.

11. Mu WD, Wang H, Zhou DS, Yu LZ, Jia TH, Li LX. Computer navigated percutaneous screw fixation for traumatic pubic symphysis diastasis of unstable pelvic ring injuries. Chin Med J(Engl). 2009;122:1699-703.

12. Sagi HC, Papp S. Comparative radiographic and clinical outcome of two-hole and multi-hole symphyseal plating. J Orthop Trauma. 2008;22:373-8.

13. Burgess AR, Eastridge BJ, Young JW, Ellison TS, Ellison Jr PS, Poka A, Bathon $\mathrm{GH}$, Brumback RJ. Pelvic ring disruptions: effective classification system and protocols. J Trauma. 1990;30:848-56.

14. Majeed SA. Grading the outcome of pelvic fractures. J Bone J Surg Br. 1989: 71:304-6.

15. Wu AM, Wang XY, Zhao HZ, Lin SL, Xu HZ, Chi YL. An imaging study of the compressed area, bony fragment area, and the total fracture involved area in thoracolumbar burst fractures. J Spinal Disord Tech. 2014;27:207-11.

16. Phieffer LS, Lundberg WP, Templeman DC. Instability of the posterior pelvic ring associated with disruption of the pubic symphysis. Orthop Clin North Am. 2004:35:445-9.

17. Meissner A, Fell M, Wilk R, Boenick U, Rahmanzadeh R. Biomechanics of the pubic symphysis. Which forces lead to mobility of the symphysis in physiological conditions? Unfallchirurg. 1996;99:415-21.
18. Walheim G, Olerud S, Ribbe T. Mobility of the pubic symphysis. Measurements by an electromechanical method. Acta Orthop Scand. 1984. 55:203-8.

19. Walheim GG, Selvik G. Mobility of the pubic symphysis. In vivo measurements with an electromechanic method and a roentgen stereophotogrammetric method. Clin Orthop Relat Res. 1984;191:129-35.

20. Cottom JM, Hyer CF, Philbin TM, Berlet GC. Treatment of syndesmotic disruptions with the Arthrex Tightrope: a report of 25 cases. Foot Ankle Int. 2008:29:773-80

21. Scheibel M, Dröschel S, Gerhardt C, Kraus N. Arthroscopic assisted stabilization of acute high-grade acromioclavicular joint separations. Am J Sports Med. 2011;39:1507-16

22. Motta P, Bruno L, Maderni A, Tosco P, Mariotti U. Acute lateral dislocated clavicular fractures: arthroscopic stabilization with TightRope. J Shoulder Elbow Surg. 2014;23:e47-52.

23. Wardle NS, Haddad FS. Proximal anterior cruciate ligament avulsion treated with Tight-Rope ${ }^{\oplus}$ fixation device. Ann R Coll Surg Engl. 2012;94:e96-8.

\section{Submit your next manuscript to BioMed Central and we will help you at every step:}

- We accept pre-submission inquiries

- Our selector tool helps you to find the most relevant journal

- We provide round the clock customer support

- Convenient online submission

- Thorough peer review

- Inclusion in PubMed and all major indexing services

- Maximum visibility for your research

Submit your manuscript at www.biomedcentral.com/submit
CioMed Central 